\title{
21st Annual Brain Impairment Conference
}

The Kevin Walsh Encouragement Award for Honours or Masters

Research was jointly awarded to Julianne Kinch and Bridget Ryburn

for the following presentations:

What Did I Have to Do Again? The Relationship Between

Neuropsychological Measures and Everyday Prospective Memory in TBI.

Julianne Kinch and Skye McDonald

University of NSW, Kensington, NSW 2033

\begin{abstract}
$\mathrm{T}$ The ability to remember to perform an intended action sometime in the future is a fundamental aspect of everyday functioning, yet it also forms one of the most common and annoying forms of memory failure. This present study investigated the effect of severe traumatic brain injury (TBI) on prospective memory. In addition, this study also sought to understand the mechanisms by which one remembers future actions, namely executive functioning and retrospective memory. Thirteen patients with severe TBI and 13 matched control subjects were assessed on two novel, yet naturalistic experimental measures of prospective memory and standard tests of neuropsychological functioning. As expected those in the TBI group performed worse than the Non-TBI group on standard retrospective memory tasks, and importantly also showed poorer performance on a time-based prospective memory task. These findings indicated that TBI affected not only retrospective but also prospective memory functioning. The effect of TBI on memory was investigated further, by examining the relative contribution of executive functioning and retrospective memory to prospective memory performance. Interestingly, results from multiple regression analyses suggested that performance on time-based prospective memory tasks (such as remembering the time of a doctor's appointment) is heavily reliant upon executive functioning (rather than retrospective memory). In addition, successful completion of event-based prospective memory tasks (for example, remembering to post a letter in the presence of a post box) was primarily attributable to retrospective memory. Hence, the results suggest that whilst prospective memory requires both executive function and retrospective memory, it appears that the ability to remember to do something at a particular time is dependent mostly upon executive functioning, whereas, the actual content of this memory is reliant upon retrospective memory. Implications of these findings for the rehabilitation of prospective memory impairment in individuals with TBI are discussed.
\end{abstract}

\section{Verbal Deficits in the Syndrome of Nonverbal Learning Disabilities: Comparing the Narrative Discourse of Children with Asperger Syndrome and Early Hydrocephalus}

Bridget Ryburn, Vicki Anderson and Roger Wales

University of Melbourne, Parkville VIC 3052

T has recently been suggested by Rourke that a variety of disorders such as Asperger Syndrome and Early Hydrocephalus can be usefully repre- 
sented by the broader category of the Syndrome of Nonverbal Learning Disabilities. Although basic linguistic skills such as pronunciation, grammar and vocabulary are described as relatively intact in children with this primarily nonverbal disability, pragmatic skills are postulated as a substantial area of weakness. The aim of this study was to empirically investigate the language of these children at several levels using a narrative discourse task, including both linguistic and more pragmatic analyses of information structure. A comprehensive neuropsychological battery was also administered. Three groups of children of average intelligence, between the ages of 8 and 14 were included in this study; 1) 10 children with uncomplicated Early Hydrocephalus, 2) 16 children with a diagnosis of Asperger Syndrome and 3) 20 healthy normal control subjects. The findings from the discourse task indicate different profiles for the two clinical groups. The results and their implication for the theory of nonverbal learning disability will be discussed.

The Luria Award for Doctoral Research was awarded to Kitty Wu for the following presentation:

\section{Specific Deficits in Attention for Attention Deficit/Hyperactivity Disorder Using the Task-Set Switching Paradigm}

Kitty Wu, Vicki Anderson and Umbersto Castiello

University of Melbourne, Parkville VIC 3052

The present study investigates the deficits of children with Attention 1 Deficit/Hyperactivity Disorder (AD/HD) in various aspects of attention. The rationale of the study is based on recent neurophysiological and neuropsychological studies that suggest frontal lobe dysfunction in AD/HD. As frontal lobe functioning is closely related to attention, AD/HD is hypothesised to be associated with specific deficits in attention. Participants were 81 children with AD/HD and 29 age-matched controls. Standardised neuropsychological tests and experimental conditions based on the task-set switching paradigm were utilised for measuring different aspects of attention. In the experiment, subjects have to switch between the colour-naming and word-reading tasks on every 2nd trial. Neutral and Stroop stimuli were presented in separate blocks of trials. Within this alternating runs paradigm, negative priming effect was manipulated for the word-reading task in the condition with Stroop stimuli. In the baseline conditions, no switching was required. Performances (i.e. accuracy, speed and variability of reaction time) in the alternating runs paradigm were compared with those in the baseline conditions. Results from experiments support the hypotheses that $\mathrm{AD} / \mathrm{HD}$ is associated with specific deficits in speed of processing and resistance for the interference of Stroop effect i.e. selective attention. The comorbidity of LD is related to deficits in switching attention and resistance for negative priming effect. Findings from standardised neuropsychological tests are consistent with the results of the experiments. In addition, the deficit associated with $\mathrm{AD} / \mathrm{HD}$ in sustained attention is identified in tests. Results are discussed in terms of current theories in task-set switching paradigm and the specific deficits of AD/HD and the co-morbidity of LD. 